O.J. GARAY

KODAI MATH. J.

11 (1988), 25-31

\title{
ON A CERTAIN CLASS OF FINITE TYPE SURFACES OF REVOLUTION
}

\author{
By OSCAR J. Garay
}

In 1983 B-Y Chen introduced the notion of Euclidean immersions of Finite Type. Essentially these are submanifolds whose immersion into $E^{m}$ is constructed by making use of a finite number of $E^{m}$-valuated eigenfunctions of their Laplacian. In terms of Finite Type terminology, a well-known result of Takahashi [9], affirms that a connected Euclidean submanifold is of 1-type, if and only if it is either minimal in $E^{m}$ or minimal in some hypersphere of $E^{m}$. So, one can consider the Finite Type submanifolds as a generalization of both minimal in $E^{m}$ and minimal into spheres. Submanifolds of Finite Type closest in simplicity to the minimal ones are the 2-type spherical submanifolds (where spherical means into a sphere). Important results about 2-type spherical closed submanifolds have been obtained in [1], [2], [3], [7].

However relatively little is known about Finite Type submanifolds which are not contained into spheres. This is so in part, because the partial differential equations which appear in the study of such submanifolds, are very difficult of dealing with. The first attempt of liberating the Finite Type submanifolds of a spherical support, was made by B-Y Chen in [4], where he proves that a non-cylindrical tube is of Infinite Type. This result along with the fact that the only closed curve of Finite Type immersed in a Euclidean space $E^{m}$, is a circle [3], incites that author to do the following conjecture [5]: The only Finite Type closed plane surface into the 3-dimensional Euclidean space is the sphere.

In this paper we want to determine the complete surfaces of revolution in $E^{3}$ whose component functions are eigenfunctions of their Laplace operator. In the first step, we shall see that this kind of surfaces are at most of 2-type. Then we shall reduce the geometric problem to a simpler ordinary differential equation system. By solving it, we obtain that a surface of this sort must be a catenoid, a right circular cylinder, or a sphere. In particular the only closed of them is the sphere, giving the first approximation to Chen's conjecture. Another consequence, is that they all have constant mean curvature. However, the mean curvature constancy is not a sufficient condition by a result of Kenmotsu, [8]. This fact is analogous to that which states that a 2-type mass-symmetric closed submanifold of $S^{n}$ has constant mean curvature, [3], but if $n=3$, then $M$ must be flat, [2], [3], and this obviously does not occur in our case. Our result can

Received July 1, 1987 
also be considered as a generalization of the well-known theorem which states that the only minimal surface of revolution in $E^{3}$ is the catenoid.

I would like to thank to $\mathrm{R}$. Ortega, for making me usefull commentaries and to the referee for his patience in revising the manuscript.

\section{Preliminaries and Examples.}

Let $x: M^{n} \rightarrow E^{m}$ be an isometric immersion of a connected Riemannian manifold $M^{n}$ into the Euclidean space $E^{m}$. We represent by $\Delta$ the Laplacian of $M^{n}$ acting on smooth functions of $M^{n}$, and by the same letter, the Laplacian acting on $E^{m}$-valuated functions. If $H$ is the mean curvature vector of the immersion $x$, we know that:

$$
\Delta x=-n H
$$

Whenever the position vector $x$ can be decomposed as a finite sum of $E^{m}$ valuated eigenfunctions of $M$ we shall say that $(M, x)$ is of Finite Type. Otherwise, we shall call it of Infinite Type. Being of Finite Type means that we are constructing the immersion $x$ by making use of a finite number of eigenvalues of $M^{n}$. As we have said, a 1-type Euclidean submanifold is either minimal in $E^{m}$ or minimal is some hypersphere of $E^{m}$. We have studied properties of Finite Type submanifolds whose image under $x$ is contained into a sphere (spherical submanifolds), but now our focus of interest are those Finite Type submanifolds not necessarily included into spheres.

If $\left(M^{n}, d s^{2}\right)$ is a $n$-dimensional Riemannian manifold with Laplacian $\Delta$, we can write locally for each $u \in C^{\infty}\left(M^{n}\right)$ :

$$
\Delta u=-\frac{1}{\sqrt{g}} \sum_{\imath, j}\left(\frac{\partial}{\partial x_{\imath}} \sqrt{g} g^{\imath \jmath} \frac{\partial}{\partial x_{\jmath}} u\right)
$$

where $d s^{2}=\sum_{i, j} g_{\imath j} d x_{i} d x_{j} ;\left(g^{i j}\right)=\left(g_{i j}\right)^{-1}$ and $g$ is the determinant of $\left(g_{i j}\right)$. We denote by $\operatorname{Spec}\left(M^{n}\right)$, and call it spectrum of $M^{n}$, the set of eigenvalues of $\Delta$. It is well known that if $M^{n}$ is compact, then $\operatorname{Spec}\left(M^{n}\right)$ is discrete and forms a not bounded sequence of non-negative eigenvalues. If $M^{n}$ is not compact, the spectrum may be purely continuous (Euclidean or Hyperbolic spaces), purely discrete, or a mixture of both types.

In this paper we shall be concerned with surfaces of revolution in $E^{3}$, which are connected, complete, and whose component functions in $E^{3}$ are eigenfunctions of their laplacians. As another consequence of our result, the only of such surfaces with discrete spectrum is the sphere, because it will be the only with finite area.

Let us represent by $\alpha(t)=(f(t), o, g(t)) ; t \in I$; any $C^{2}$ curve in $x z$-plane, whose domain of definition $I$ is any open real interval. A surface of revolution of $E^{3}$ is defined by: 


$$
M=\{(f(t) \cos \theta, f(t) \sin \theta, g(t))\} ; t \in I ; 0 \leqq \theta \leqq 2 \pi .
$$

If we suppose that this surface of revolution is constructed with component functions which are eigenfunctions of its Laplacian, we shall have that $\Delta(f \cos \theta)$ $=\lambda_{1} f \cos \theta ; \Delta(f \sin \theta)=\lambda_{2} f \sin \theta ;$ and $\Delta g=\mu g ; \lambda_{1}, \lambda_{2}, \mu \in \operatorname{Spec}(M)$. This means that $M$ is at most of 3-type.

By the regularity of $M$, we can take $f(t)>0 ; t \in I$. Then if one uses formula (2), we obtain for $u \in C^{\infty}(M)$ :

$$
\Delta u=-\left\{\frac{\left[\log \frac{f}{\left(\left(f^{\prime}\right)^{2}+\left(g^{\prime}\right)^{2}\right)^{1 / 2}}\right]}{\left(f^{\prime}\right)^{2}+\left(g^{\prime}\right)^{2}} \frac{\partial u}{\partial t}+\frac{1}{\left(f^{\prime}\right)^{2}+\left(g^{\prime}\right)^{2}} \frac{\partial^{2} u}{\partial t^{2}}+\frac{1}{f^{2}} \frac{\partial^{2} u}{\partial \theta^{2}}\right\}
$$

Thus, $f(t) \cos \theta$ and $f(t) \sin \theta$, are eigenfunctions of $\Delta$ for $\lambda_{1} ; \lambda_{2}$ respectively, if and only if, $f(t)$ satisfies:

$$
\begin{aligned}
& \frac{\left(f^{\prime}\right)^{2}}{f} \frac{1}{\left(f^{\prime}\right)^{2}+\left(g^{\prime}\right)^{2}}-\frac{f^{\prime} f^{\prime \prime}+g^{\prime} g^{\prime \prime}}{\left(\left(f^{\prime}\right)^{2}+\left(g^{\prime}\right)^{2}\right)^{2}} f^{\prime}+\frac{f^{\prime \prime}}{\left(f^{\prime}\right)^{2}+\left(g^{\prime}\right)^{2}}-\frac{1}{f}=-\lambda_{1} f \\
& \frac{\left(f^{\prime}\right)^{2}}{f} \frac{1}{\left(f^{\prime}\right)^{2}+\left(g^{\prime}\right)^{2}}-\frac{f^{\prime} f^{\prime \prime}+g^{\prime} g^{\prime \prime}}{\left(\left(f^{\prime}\right)^{2}+\left(g^{\prime}\right)^{2}\right)^{2}} f^{\prime}+\frac{f^{\prime \prime}}{\left(f^{\prime}\right)^{2}+\left(g^{\prime}\right)^{2}}-\frac{1}{f}=-\lambda_{2} f
\end{aligned}
$$

and then $\lambda_{1}=\lambda_{2}$ and we shall call it symply $\lambda$ from now on. This means that $M$ is at most of 2-type.

Similarly, $g(t)$ is an eigenfunction with eigenvalue $\mu$ if and only if,

$$
\frac{f^{\prime} g^{\prime}}{f} \frac{1}{\left(f^{\prime}\right)^{2}+\left(g^{\prime}\right)^{2}}-\frac{f^{\prime} f^{\prime \prime}+g^{\prime} g^{\prime \prime}}{\left(\left(f^{\prime}\right)^{2}+\left(g^{\prime}\right)^{2}\right)^{2}} g^{\prime}+\frac{g^{\prime \prime}}{\left(f^{\prime}\right)^{2}+\left(g^{\prime}\right)^{2}}=-\mu g
$$

We shall see now some examples.

i) Catenoid

We consider $x(t, \theta)=(a \cos h(t) \cos \theta, a \cos h(t) \sin \theta, a t) ; 0 \leqq \theta \leqq 2 \Pi-\infty<t<$ $\infty$. In this case $f(t)=a \cos h t$ and $g(t)=a t$, and they verify (4) and (5) if and only if $\lambda=\mu=0$. So it is minimal in $E^{3}$.

ii) Sphere

We take $x(t, \theta)=(\sin t \cos \theta, \sin t \sin \theta, \cos t) ; 0<t<\Pi ; 0 \leqq \theta \leqq 2 \Pi$. Now $f(t)$ $=\sin t$ and $g(t)=\cos t$, and they verify (4) and (5) for $\lambda=\mu=2$. This means $\left(S^{2}, x\right)$ is of 1-type.

\section{iii) Cylinder}

We take $x(t, \theta)=(r \cos \theta, r \sin \theta, t)$ for some $r>0$ and $t$ varying between $-\infty$ and $\infty$. Hence, $f(t)=r$ and $g(t)=t$. They verify (4) and (5) for $\mu=0$ and $\lambda=1 / r^{2}$. So the right circular cylinder is of 2-type.

iv) Of course not every element of the set of surfaces of revolution is of Finite Type. For instance, a embedded torus in $E^{3}$ is of Infinite Type, [4]. 


\section{Our result.}

For the sake of simplicity, we suppose without loss of generality that the curve $\alpha$ is parametrized by the arc length. So

$$
\left(f^{\prime}\right)^{2}+\left(g^{\prime}\right)^{2}=1
$$

With this condition, equations (4) and (5) turns into:

$$
\begin{aligned}
& \frac{\left(f^{\prime}\right)^{2}}{f}+f^{\prime \prime}-\frac{1}{f}=-\lambda f \\
& \frac{f^{\prime} g^{\prime}}{f}+g^{\prime \prime}=-\mu g
\end{aligned}
$$

We distinguish three posibilities according to $\lambda$ is lesser, equal, or greater than zero.

Case one: $\lambda<0$

In this case we can use equations (6) and (7) to obtain:

$$
K=\lambda-\left\{\frac{g^{\prime}}{f}\right\}^{2}
$$

where $K$ is the Gauss curvature of $M$. But it means that $K \leqq \lambda<0$ and this is imposible by a result of Efimov [6]. So $\lambda<0$ can not occur.

Case two: $\lambda=0$

If $\lambda=0$ equation (7) gives:

$$
\left(f^{\prime}\right)^{2}+f f^{\prime \prime}=1
$$

or, equivalently

$$
\frac{1}{2} \frac{d^{2} f^{2}}{d t^{2}}=1
$$

and then

$$
f(t)=\left(t^{2}+k_{1} t+k_{2}\right)^{1 / 2}
$$

with $k_{1}^{2}-4 k_{2} \leqq 0 ; k_{1}, k_{2} \in R$.

From (6) and (11), we obtain

$$
\begin{aligned}
& f^{\prime}=\frac{1}{2}\left\{2 t+k_{1}\right\}\left\{t^{2}+k_{1} t+k_{2}\right\}^{-1 / 2} \\
& g^{\prime}=\left\{1-\frac{\left(2 t+k_{1}\right)^{2}}{4\left(t^{2}+k_{1} t+k_{2}\right)}\right\}^{1 / 2}
\end{aligned}
$$

and consequently 


$$
g^{\prime \prime}=-\frac{2 t+k_{1}}{2\left(t^{2}+k_{1} t+k_{2}\right)}\left\{1-\frac{\left(2 t+k_{1}\right)^{2}}{4\left(t^{2}+k_{1} t+k_{2}\right)}\right\}^{1 / 2}
$$

Putting this equations in (8) we obtain that $\mu$ is also zero. Then equation (1) gives that $M$ is minimal, and so, it is the catenoid. (Another considerations of sign in (13) lead to the same result).

Case three. $\lambda>0$

In this last case we have $x(t, \theta)=(f \cos \theta, f \sin \theta, g)$ with $f>0$, and $f^{\prime} g^{\prime} \neq 0$ because $M$ is regular. Calling $x_{1}=(f \cos \theta, f \sin \theta, 0)$ and $x_{2}=(0,0, g)$, we have $\Delta x_{1}=\lambda x_{1}$ and $\Delta x_{2}=\mu x_{2}$, where $\lambda>0$.

Now equation (7) says that

$$
\frac{1}{2} \frac{d^{2} f^{2}}{d t^{2}}-1=-\lambda f^{2}
$$

and integrating this equation, we obtain without loss of generality by the election of signs:

$$
f(t)=\left\{\frac{1+\left\{2 \lambda\left(k_{1}+\frac{1}{2 \lambda}\right)\right\}^{1 / 2} \cos \left(\sqrt{2 \lambda}\left(t+k_{2}\right)\right)}{\lambda}\right\}^{1 / 2}
$$

where $k_{1}$ and $k_{2}$ are constants. We call $A=\sqrt{2 \lambda}$ and $B=\sqrt{k_{1}+(1 / 2 \lambda)}$. We also can suppose that $k_{2}=0$, and then:

$$
f(t)=\frac{\sqrt{2}}{A}\{1+A B \cos (A t)\}^{1 / 2}
$$

Directly from (17) and (16) we get

$$
\begin{gathered}
f^{\prime}(t)=-\frac{A B}{\sqrt{2}} \sin (A t)\{1+A B \cos (A t)\}^{-1 / 2} \\
g^{\prime}(t)=\left\{1-\frac{A^{2} B^{2}}{2} \frac{\sin ^{2}(A t)}{1+A B \cos (A t)}\right\}^{1 / 2} \\
g^{\prime \prime}(t)=-\frac{A^{3} B^{2}}{4} \frac{\sin (A t)}{(1+A B \cos (A t))^{2}} \frac{2 \cos (A t)+A B\left(1+\cos ^{2}(A t)\right)}{\left\{1-\frac{A^{2} B^{2} \sin ^{2}(A t)}{2(1+A B \cos (A t))}\right\}^{1 / 2}}
\end{gathered}
$$

By using equations (17) to (20) together with equation (8) and making a long computation, we obtain that $\mu g$ must be

$$
\mu g(t)=\frac{A^{2} B}{2} \frac{\sin (A t)}{\left\{1-\frac{A^{2} B^{2} \sin ^{2}(A t)}{2(1+A B \cos (A t))}\right\}^{1 / 2}}
$$

From this formula we obtain: 


$$
\mu g^{\prime}=\frac{\frac{A^{3} B}{4}\left\{\frac{A^{3} B^{3} \sin ^{4}(A t)}{2(1+A B \cos (A t))^{2}}+2 \cos (A t)\right\}}{\left\{1-\frac{A^{2} B^{2} \sin ^{2}(A t)}{2(1+A B \cos (A t))}\right\}^{3 / 2}}
$$

and by using (22) and (19), we get:

$$
\mu=\frac{\frac{A^{3} B}{4}\left\{\frac{A^{3} B^{3} \sin ^{4}(A t)}{2(1+A B \cos (A t))^{2}}+2 \cos (A t)\right\}}{\left\{1-\frac{A^{2} B^{2} \sin ^{2}(A t)}{2(1+A B \cos (A t))}\right\}^{2}}
$$

and remembering that $\mu$ is constant, this is true when

$$
\begin{aligned}
& \frac{A^{3} B}{2}\left\{A^{3} B^{3} \sin ^{4}(A t)+4 \cos (A t)(1+A B \cos (A t))^{2}\right\} \\
& =\mu\left\{2(1+A B \cos (A t))-A^{2} B^{2} \sin ^{2}(A t)\right\}^{2}
\end{aligned}
$$

One can verify that this equation holds, if and only if, one of the following cases is true: i) $A B=0$; ii) $A B=1$; iii) $A B=-1$.

If $A B=0$, then $\mu=0$ and from (17) and (19) we obtain $f(t)=(1 / \sqrt{\lambda}) ; g(t)=$ $t+k_{3}$, with $k_{3}$ constant, and so $M$ is a right circular cylinder. If $A B=1$, we use the definitions of $A$ and $B$ to get $k_{1}=0$, and consequently from (16)

$$
f(t)=\sqrt{\frac{2}{\lambda}} \cos \left(\sqrt{\frac{\lambda}{2}} t\right) ; g(t)=\sqrt{\frac{2}{\lambda}} \sin \left(\sqrt{\frac{\lambda}{2}} t\right)
$$

and (8) gives $\mu=\lambda$. Thus we have a sphere.

Finally, $A B=-1$ is impossible by our election of $A$ and $B$. Another considerations of signs lead to the same conclusions.

In short, we have proved

THEOREM. Let $M$ be a complete surface of revolution in $E^{3}$, whose component functions are eigenfunctions of its Laplacian, then the surface must be a catenoid, $a_{\Perp}^{\mathbf{7} p h e r e}$ or a right circular cylinder.

This result leads immediatly to the following corollary:

COROLLARY. Under above conditions, a surface of revolution in $E^{3}$ must be either:

1) minimal in $E^{3}$, and so it is the catenoid; or

2) of 1-type not minimal, and then it is the sphere $S^{2}$; or

3) of 2-type in $E^{3}$, and in this case it will be the right circular cylinder.

As a consequence of this corollary, the sphere is the only compact surface of revolution whose component functions are eigenfunctions of its Laplacian, and therefore is the only closed surface of revolution of this kind which can 
be of Finite Type. This gives a partial answer to Chen's conjecture.

\section{REFERENCES}

[1] M. Barros And B-Y. Chen, Stationary 2-type surfaces in a hypersphere, To appear in J. Math. Soc. Japan.

[2] M. Barros and O.J. Garay, 2-type surfaces in $S^{3}$, Geometriae Dedicata, 24 (1987), 329-336.

[3] B-Y. CHEN, Total mean curvature and submanifolds of Finite Type, World Scientific, Singapore, 1984.

[4] B-Y. Chen, Surfaces of Finite Type in $E^{3}$, Preprint.

[5] B-Y. CHEN, Finite submanifolds and generalizations, Quaderni dil seminari di Geometria Algebraica e Dif. Univ. Studi di Roma, 1985.

[6] N. EFImov, Appearence of singularities on surfaces of negative curvature, Math. Sb., 106, 1954.

[7] O.J. Garay, Superficies esféricas de tipo dos, Tesis de la Universidad de Granada, 1987.

[8] K. Kenmotsu, Surfaces of revolution with precribed mean curvature, Tohoku Math. J., 32 (1980), 147-153.

[9] T. TAKAHASHI, Minimal immersions of Riemannian manifolds, J. Math. Soc. Japan, 18 (1966), 380-385.

Departamenta de Geometría y Topología

Facultad de Ciencias

Av. Fuentenueva $\mathrm{s} / \mathrm{n}$

18002, Granada, Spain 